Research Article

\title{
COVID on the Brain: Anxiety Sensitivity and Mindful Awareness Mediate the Relationship between COVID-19 Obsession and Anxiety
}

\author{
Emily Beitzell, Purnaja Podduturi, Megan Hook, Amelia Jones, Haley McKeen, Miriam Liss *
}

Department of Psychological Science, University of Mary Washington, DC, USA; E-Mails: ebeitzel@umw.edu; ppoddutu@mail.umw.edu; mhook@mail.umw.edu; ajones29@umw.edu; hmckeen@umw.edu; mliss@umw.edu

* Correspondence: Miriam Liss; E-Mail: mliss@umw.edu

Academic Editor: Gerhard Litscher

OBM Integrative and Complementary Medicine 2021, volume 6 , issue 4

doi:10.21926/obm.icm.2104053
Received: October 03, 2021

Accepted: November 29, 2021

Published: December 01, 2021

\begin{abstract}
The coronavirus (COVID-19) pandemic has produced a global mental health crisis and many individuals have obsessive thoughts and worry about COVID-19 which may, both directly and indirectly, relate to more general anxiety. This study explored anxiety sensitivity and mindful awareness as mediators in the relationship between COVID-19 obsession and general anxiety. College student participants $(N=172)$ completed the Obsession with COVID-19 scale, the Anxiety Sensitivity Index, the awareness subscale of the Five Factor Mindfulness Questionnaire, and the Generalized Anxiety Disorder Scale. Process was used to test a mediational model. We found both anxiety sensitivity and mindful awareness to be significant mediators such that COVID-19 obsession predicted increased anxiety sensitivity which predicted decreased mindful awareness which, in turn, predicted increased general anxiety. These results demonstrate that anxiety about COVID-19 increases awareness and worry surrounding one's physical and mental state which lead to a decrease in mindful awareness. Our findings identify mechanisms by which worry about COVID-19 may be leading to increased general anxiety and can be used to inform mindfulness interventions to help prevent these negative effects.
\end{abstract}

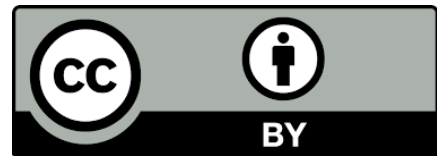

(C) 2021 by the author. This is an open access article distributed under the conditions of the Creative Commons by Attribution License, which permits unrestricted use, distribution, and reproduction in any medium or format, provided the original work is correctly cited. 


\section{Keywords}

COVID-19; anxiety; anxiety sensitivity; mindfulness; mediation

\section{Introduction}

The Coronavirus (COVID-19) pandemic has forced unprecedented changes in behavior globally. A study conducted in China showed that almost a third of the population has experienced psychological distress since the beginning of the pandemic [1]. Quarantine mandates worldwide have produced nonspecific and uncontrollable fears related to infection, pervasive anxiety, frustration and boredom, disabling loneliness, and in some cases suicidal behaviors [2, 3]. Although vaccines are available, the rise of the Delta variant has kept concerns about COVID-19 in the forefront of many people's minds and mental health concerns continue to rise [4]. Research has shown elevated levels of anxiety symptoms during the pandemic [5], especially among those with pre-existing anxiety related and mood disorders [6]. Given the anxiety provoking environment COVID-19 is creating, it is important to understand the mechanisms by which living with COVID-19 may predict anxiety. In this study we will explore the constructs of anxiety sensitivity and mindful awareness to determine whether they serve as mediators in the relationship between obsessive thoughts about COVID-19 and more general symptoms of anxiety.

Research has identified some of the main concerns about COVID-19 to be the health of others, healthcare collapse, consequences for the economy, personal health, and mass panic [7]. All of these factors are leading to obsessive thoughts about COVID-19, such as worrying about contracting the virus, having dreams about it, and disturbing thoughts that others may have the virus [8]. These obsessive thoughts are common symptoms of clinical anxiety and are strongly associated with coronavirus anxiety, alcohol/drug coping, extreme helplessness, and suicidal ideation [8]. While COVID-19 anxiety has been looked at in relation to overall mental health, the mechanisms by which this specific type of anxiety is linked to more general anxiety have not been fully explored.

Given that COVID-19 is a physical illness with a wide variety of possible symptoms, it may increase our tendency to notice and be concerned about our physical states. Anxiety sensitivity involves the fear of arousal-related sensations and the belief that these sensations will cause social or physical consequences, and is positively related to anxiety and anxiety disorders [9]. Research has shown that fear of COVID-19 is related to higher levels of anxiety sensitivity [10]. Worry about COVID-19 may enhance an individual's tendency to think that every cough or sneeze means they have the virus, even if there is no actual evidence that they do. Thus, anxiety sensitivity may be a mechanism by which obsessive thoughts about COVID-19 may result in more general anxiety.

Anxiety over COVID-19 and hyper attention to one's bodily states may also decrease a person's ability to pay attention to and live in the present moment. In other words, it may decrease one's level of mindful awareness. Mindful awareness has been correlated with experiencing less psychological distress during the COVID-19 pandemic [4], as well as decreased anxiety, depression, emotional exhaustion, and increased psychological awareness during quarantine mandates [11]. Saricali et al. [12] found mindful awareness to be an important factor in regulating fear of COVID19 and reducing hopelessness. It is possible that individuals are becoming so concerned with the internal workings of their bodies and worrying about the virus that they are less aware of the world 
around them. Furthermore, there is evidence that anxiety sensitivity is related to lower levels of mindfulness $[13,14]$. Overall, these findings support the idea that increased anxiety sensitivity and decreased mindful awareness may be mechanisms by which COVID-19 obsession results in more generalized anxiety. Specifically, we hypothesize that increased anxiety sensitivity and decreased mindful awareness will mediate the relationship between obsession with COVID-19 and more generalized anxiety.

\section{Method}

\subsection{Participants}

A total of 172 individuals attending a public liberal arts college located in the Southeast United States participated in this study. Participants ranged from 17 to 48 years of age, with an average age of 19.13 (SD =2.90). The majority of participants identified as White $(69.8 \%, n=120), 11.0 \%$ identified as Black/African American ( $n=19), 8.1 \%$ identified as Latinx $(n=14), 3.5 \%$ identified as multiracial $(n=6), 3.5 \%$ identified as East Asian $(n=6), 1.7 \%$ identified as South Asian $(n=3), 0.6 \%$ identified as Middle Eastern $(n=1), 0.6 \%$ identified as Native Hawaiin/Pacific Islander $(n=1), 0.6 \%$, and $1.2 \%$ identified as other $(n=2)$. The majority of participants were first year students $(66.3 \%, n$ $=114), 18 \%$ were second year students $(n=31), 9.9 \%$ were third year students $(n=17), 3.5 \%$ were fourth year students $(n=6)$, and $1.2 \%$ were fifth year students $(n=2)$. Most participants identified as female $(79.1 \%, n=136), 20.3 \%$ identified as male $(n=35)$, and $0.6 \%$ identified as other $(n=1)$.

\subsection{Procedures and Measures}

This study was approved by the Institutional Review Board at a Southeastern liberal arts college. Participants were recruited using the General Psychology participant pool and this study was conducted as an online survey. All participants read an informed consent page and indicated consent by clicking to continue with the survey. At the end of the survey, all participants were debriefed and provided with mental health resources, if needed. In addition to demographics, participants were given the following measures.

\subsubsection{Mindful Awareness}

The Acting with Awareness subscale of the Five Factor Mindfulness Questionnaire (FFMQ; Baer et al. [15]) is an eight-item questionnaire scored on a scale from 1 (never/rarely true of me) to 5 (very often/always true of $m e$ ) and exhibited good reliability $(\alpha=.89)$.

\subsubsection{Obsession with COVID-19}

The Obsession with COVID-19 Scale [8] is a four-item measure scored on a scale from 0 (not at all) to 4 (nearly every day over the last 2 weeks) and exhibited good reliability $(a=.77)$.

\subsubsection{Anxiety Sensitivity}

The Anxiety Sensitivity Index [9] is a 16-item self-report measure scored on a scale from 0 (very little) to 4 (very much) and exhibited good reliability $(a=.91)$. 


\subsubsection{Generalized Anxiety Disorder Scale (GAD)}

The General Anxiety Disorder Scale (GAD-7; Spitzer et al. [16]) is a seven-item measure scored on a scale of 1 (not at all) through 4 (nearly every day) and exhibited good reliability $(\alpha=0.93$ ).

\section{Results}

Descriptive statistics and correlations among all major measures are reported in Table 1. According to the GAD-7 cutoff scores, $35.2 \%$ of our sample was mildly anxious, $16.4 \%$ was moderately anxious, and $19.8 \%$ was severely anxious [16]. Additionally, $10.5 \%$ met the criteria for dysfunctional thinking about COVID-19 [8].

Table 1 Descriptive statistics and correlations among all measures.

\begin{tabular}{|c|c|c|c|c|c|c|c|c|}
\hline \multirow{2}{*}{ Measures } & \multirow{2}{*}{$M$} & \multirow{2}{*}{$S D$} & \multirow{2}{*}{$\begin{array}{l}\text { Actual } \\
\text { Range }\end{array}$} & \multirow{2}{*}{$\begin{array}{l}\text { Possible } \\
\text { Range }\end{array}$} & \multicolumn{4}{|c|}{ Correlation } \\
\hline & & & & & 1 & 2 & 3 & 4 \\
\hline 1. COVID-19 Obsession & 3.35 & 2.96 & $0-14$ & $0-16$ & - & & & \\
\hline $\begin{array}{l}\text { 2. Anxiety Sensitivity } \\
\text { (ASI) }\end{array}$ & 26.80 & 13.89 & $3-64$ & $0-64$ & $.45 * *$ & - & & \\
\hline $\begin{array}{l}\text { 3. Mindful Awareness } \\
\text { (FFMQ) }\end{array}$ & 2.76 & .89 & $1-5$ & $1-5$ & $-.25 * *$ & $-.41 * *$ & - & \\
\hline 4. Anxiety (GAD) & 9.04 & 6.38 & $0-21$ & $0-28$ & $.32 * *$ & $.65^{* *}$ & $-.53 * *$ & - \\
\hline
\end{tabular}

Note. ${ }^{* *} p \leq .001$.

All variables were significantly correlated in the expected directions. Specifically, COVID-19 obsession was positively correlated with anxiety sensitivity and anxiety. Anxiety sensitivity was positively correlated with anxiety. Mindful awareness was negatively correlated with COVID-19 obsession, anxiety sensitivity, and anxiety.

To test our hypothesis, we analyzed how levels of anxiety sensitivity and mindful awareness mediated the relationship between obsession with COVID-19 and general anxiety using PROCESS (Hayes [17]; Model 6). Anxiety sensitivity and mindful awareness significantly mediated the relationship between COVID-19 obsession and anxiety and had a complete standardized indirect effect of $.05(S E=.02, \mathrm{Cl}=[.02, .09])$. This model explained $50.2 \%$ of the variance in anxiety scores, $F(1,170)=46.29, p<.001$ (see Figure 1 for path coefficients). There was also a statistically significant three-variable indirect effect. The relationship between COVID-19 obsession and general anxiety was significantly mediated by anxiety sensitivity, with a completely standardized effect of .23 ( $S E=$ $.05, \mathrm{Cl}=[.14, .32])$. 


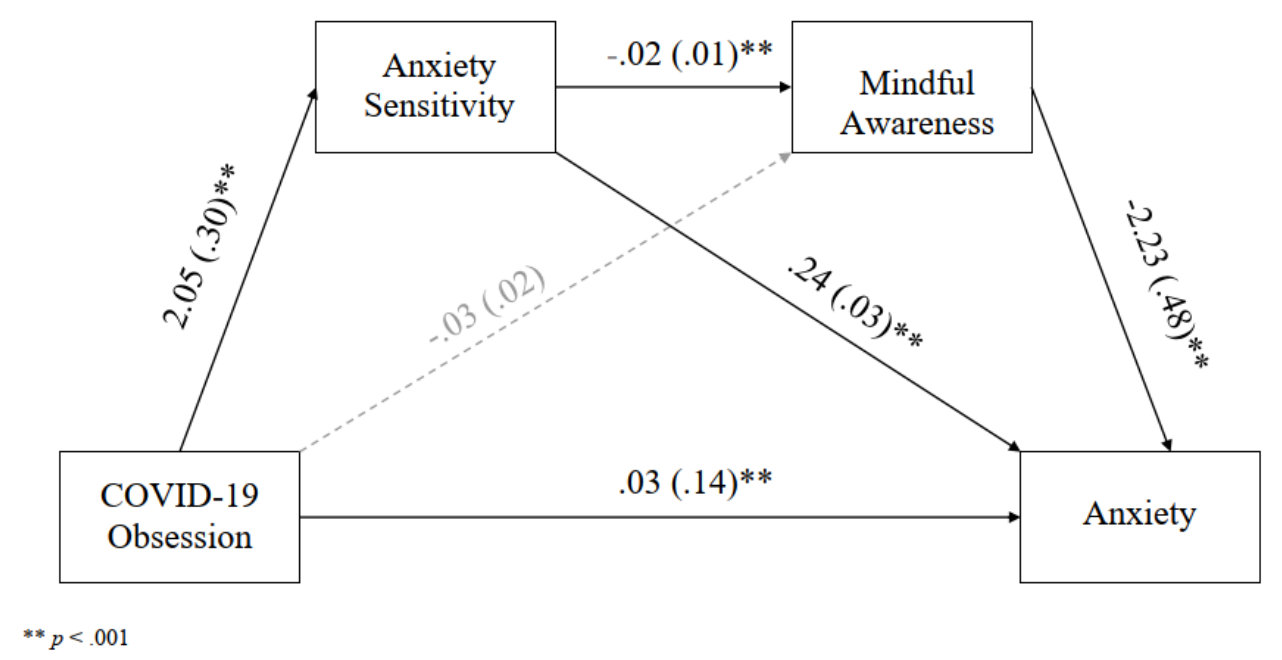

Figure 1 Path coefficients (and standard errors) showing the relationship between COVID-19 obsession and anxiety mediated by anxiety sensitivity and mindful awareness.

\section{Discussion}

The goal of our study was to examine which mechanisms may explain the relationship between COVID-19 obsession and generalized anxiety. We hypothesized that higher levels of anxiety sensitivity and lower levels of mindful awareness would mediate the relationship between COVID19 obsession and anxiety. Overall, $36.2 \%$ of our sample was moderately to severely anxious at the time of data collection. This demonstrates the level of anxiety felt during the COVID-19 pandemic, similar to previous findings $[5,6]$. We found that anxiety sensitivity and mindful awareness both mediated the relationship between COVID-19 obsession and anxiety. Our findings align with previous research on anxiety sensitivity and mindful awareness during the pandemic suggesting that fear of COVID-19 is related to high levels of anxiety sensitivity [10], as well as research that shows that anxiety sensitivity is related to low mindful awareness [13].

Anxiety about COVID-19 may be increasing the level of anxiety sensitivity due to increased worry surrounding bodily states. Individuals may obsessively monitor for symptoms and wrongfully assume every cough or sneeze they have means they have been infected with the virus, as well as, worry that others around them have contracted the virus. Further, anxiety sensitivity also assesses worry about mental states; individuals may also be concerned about the level of worry they are experiencing and compare their struggles to those of others, in turn increasing the intensity of anxiety they feel. Thus, worry about COVID-19 appears to increase the attention that individuals are paying both to their physical and mental states.

This increase in anxiety sensitivity is related to a decrease in mindful awareness. When an individual spends so much time concerned with their body, caught up in thoughts about the virus, and completely distracted from daily life, they are less capable of being aware of the world around them. This decrease in mindful awareness is related to more general symptoms of anxiety as people may be worrying about their mental state instead of living in the present moment. Furthermore, there may be a negative feedback loop where individuals are aware that they are not paying attention to their surroundings and are overly worried about COVID-19, which may increase their anxiety about their mental state. Thus, while we modeled anxiety sensitivity as leading to decreased 
mindfulness, it is also likely that decreased mindfulness, and concern over one's lack of attention to day to day life may be increasing anxiety sensitivity. We did explore a model in which we reversed the mediators and mindfulness to predict anxiety sensitivity. We found very similar findings and a similar indirect effect between COVID-19 obsession and general anxiety. Thus, these two variables are likely bidirectional.

\subsection{Limitations}

These findings should be interpreted in the context of the study's limitations. First, the present model could be bidirectional. We only tested the model in one direction in which obsession with COVID-19 leads to anxiety mediated by anxiety sensitivity and mindful awareness. However, as noted this model appears to be bidirectional; further, both of these may be influenced by general anxiety in a negative feedback loop. Further, temperamentally anxious people may be more likely to experience COVID-19 related worries in the first place. However, we explored a reverse model in which general anxiety was modeled to predict COVID-19 related worry and this model did not have a significant indirect effect. Second, we used an older version of the Anxiety Sensitivity Index [9] that did not divide anxiety sensitivity into factor scores. If we used the most recent version, we may have found that physical anxiety sensitivity is particularly problematic. However, using the more general measure demonstrates that anxiety sensitivity in general is an important mechanism. Third, our sample was demographically homogeneous, consisting of predominantly white educated women. This makes it difficult when generalizing our findings to other populations. Fourth, data collection took place in October and November of 2020. Concerns surrounding COVID-19 are constantly evolving and the situation has changed significantly since data collection, especially since the development of vaccines. However, this does not mean that anxiety regarding the pandemic has disappeared.

Worry about COVID-19 and the consequences of this worry may also be influenced by other variables that were not measured in the current study. For example, data from China has suggested that individuals with a greater sense of community and with more economic resources are less worried while those who live with large families are more worried [18]. It is also important to look beyond anxiety to other outcomes such as life satisfaction. Research has demonstrated that the stresses of COVID-19 are considerably more than the fear of actually catching the disease and include stress about job security, social isolation, and the overall stress to changes in the way to life [19]. In fact, the physical worries about getting the virus may be less concerning than these other issues and one study found that those with high fears of getting the virus and high stress were generally more satisfied with their lives than those with lower fears of getting COVID-19 [19]. Thus, anxiety and obsession about COVID-19 should also be considered within the context of other stressors associated with the life disruption of COVID-19.

\section{Conclusion}

These findings have important clinical implications. Our findings demonstrate that those with high levels of anxiety sensitivity may be at the highest risk for negative mental effects from the circumstances surrounding this pandemic and future pandemics. Having high levels of mindful awareness may also be protective. Mindful awareness may enable individuals to look past the anxiety surrounding the virus and may be protected from negative effects of worry about COVID- 
19. This study adds to a small body of literature that has identified mindful awareness as playing an important role in managing anxiety during this global pandemic [4, 11, 12]. Implementing mindfulness interventions could be beneficial in preventing future mental health crises during the current and any future pandemics. Overall, our research contributes to the literature on mental health concerns during the COVID-19 pandemic and increases our understanding of the mechanisms by which worry about COVID-19 leads to anxiety.

\section{Author Contributions}

ML supervised this study and EB did the majority of the writing. The remaining four authors participated in the conceptualization of the study, data collection, data analysis, and assisted with writing.

\section{Funding}

This research did not receive funding from any organizations or foundations.

\section{Competing Interests}

The authors have declared that no competing interests exist.

\section{References}

1. Qiu J, Shen B, Zhao M, Wang Z, Xie B, Xu Y. A nationwide survey of psychological distress among Chinese people in the COVID-19 epidemic: Implications and policy recommendations. Gen Psychiatry. 2020; 33: e100213.

2. Serafini G, Parmigiani B, Amerio A, Aguglia A, Sher L, Amore M. The psychological impact of COVID-19 on the mental health in the general population. QJM Int J Med. 2020; 113: 531-537.

3. Thakur V, Jain A. COVID 2019-suicides: A global psychological pandemic. Brain Behav Immun. 2020; 88: 952.

4. Conversano C, Di Giuseppe M, Miccoli M, Ciacchini R, Gemignani A, Orrù G. Mindfulness, age and gender as protective factors against psychological distress during COVID-19 pandemic. Front Psychol. 2020; 11: 1900.

5. Liu CH, Zhang E, Wong GT, Hyun S. Factors associated with depression, anxiety, and PTSD symptomatology during the COVID-19 pandemic: Clinical implications for US young adult mental health. Psychiatry Res. 2020; 290: 113172.

6. Asmundson GJ, Paluszek MM, Landry CA, Rachor GS, McKay D, Taylor S. Do pre-existing anxietyrelated and mood disorders differentially impact COVID-19 stress responses and coping? J Anxiety Disord. 2020; 74: 102271.

7. Mertens G, Gerritsen L, Duijndam S, Salemink E, Engelhard IM. Fear of the coronavirus (COVID19): Predictors in an online study conducted in March 2020. J Anxiety Disord. 2020; 74: 102258.

8. Lee SA. How much "Thinking" about COVID-19 is clinically dysfunctional? Brain Behav Immun. 2020; 87: 97-98.

9. Reiss S, Peterson RA, Gursky DM, McNally RJ. Anxiety sensitivity, anxiety frequency and the prediction of fearfulness. Behav Res Ther. 1986; 24: 1-8. 
10. McKay D, Yang H, Elhai J, Asmundson GJ. Anxiety regarding contracting COVID-19 related to interoceptive anxiety sensations: The moderating role of disgust propensity and sensitivity. J Anxiety Disord. 2020; 73: 102233.

11. Matiz A, Fabbro F, Paschetto A, Cantone D, Paolone AR, Crescentini C. Positive impact of mindfulness meditation on mental health of female teachers during the COVID-19 outbreak in Italy. Int J Environ Res Public Health. 2020; 17: 6450.

12. Saricali M, Satici SA, Satici B, Gocet-Tekin E, Griffiths MD. Fear of COVID-19, mindfulness, humor, and hopelessness: A multiple mediation analysis. Int J Ment Health Addict. 2020. Doi: 10.1007/s11469-020-00419-5.

13. McKee L, Zvolensky MJ, Solomon SE, Bernstein A, Leen-Feldner E. Emotional-vulnerability and mindfulness: A preliminary test of associations among negative affectivity, anxiety sensitivity, and mindfulness skills. Cogn Behav Ther. 2007; 36: 91-101.

14. Waszczuk MA, Zavos HM, Antonova E, Haworth CM, Plomin R, Eley TC. A multivariate twin study of trait mindfulness, depressive symptoms, and anxiety sensitivity. Depress Anxiety. 2015; 32: 254-261.

15. Baer RA, Smith GT, Hopkins J, Krietemeyer J, Toney L. Using self-report assessment methods to explore facets of mindfulness. Assessment. 2006; 13: 27-45.

16. Spitzer RL, Kroenke K, Williams JB, Löwe B. A brief measure for assessing generalized anxiety disorder: The GAD-7. Ann Intern Med. 2006; 166: 1092-1097.

17. Hayes AF. Introduction to mediation, moderation, and conditional process analysis: A regression-based approach. 2nd ed. New York: Guilford Press; 2018.

18. Zhou M, Guo W. Social factors and worry associated with COVID-19: Evidence from a large survey in China. Soc Sci Med. 2021; 277: 113934.

19. Dymecka J, Gerymski R, Machnik-Czerwik A. Fear of COVID-19 as a buffer in the relationship between perceived stress and life satisfaction in the Polish population at the beginning of the global pandemic. Health Psychol Rep. 2021; 9: 149-159.

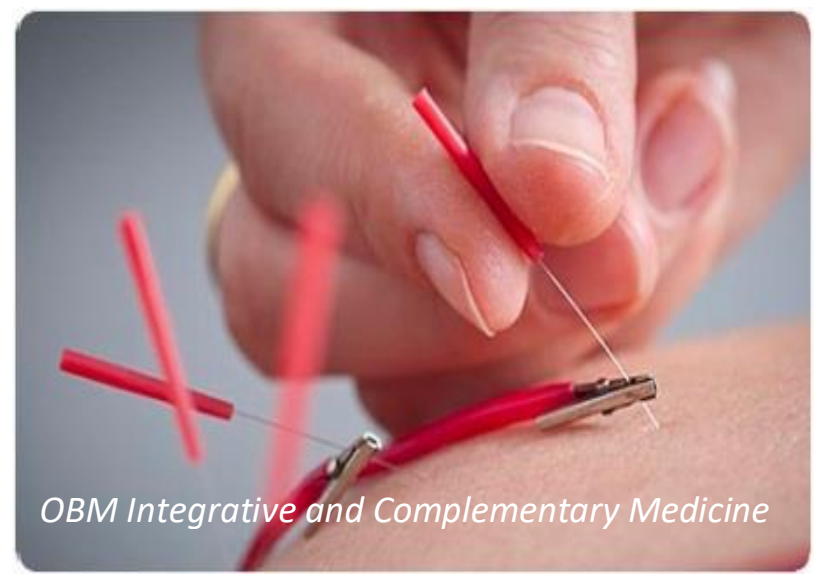

Enjoy OBM Integrative and Complementary Medicine by:

1. Submitting a manuscript

2. Joining in volunteer reviewer bank

3. Joining Editorial Board

4. Guest editing a special issue

For more details, please visit: http://www.lidsen.com/journals/icm 